\title{
Glycemic Variability in Type 1 Diabetes-Does It Matter?
}

\author{
Frank L Schwartz, MD, FACE¹ and Cynthia R Marling, PhD²
}

\author{
1. Professor of Endocrinology, The Diabetes Institute, Ohio University Heritage College of Osteopathic Medicine, Athens, Ohio, US; \\ 2. Associate Professor, School of Electrical Engineering and Computer Science, Russ College of Engineering and Technology, Ohio University, Athens, Ohio, uS
}

\begin{abstract}
Clinical assessment of glycemic variability (GV) attempts to measure factors that may be contribute to tissue damage and the complications of diabetes that are not measured in glycosylated hemoglobin $\left(\mathrm{HbA}_{1 \mathrm{c}}\right)$. Physicians managing patients with diabetes immediately understand the concept of GV; however, how it is assessed in clinical research trials and whether it has any predictive power in patients with type 1 diabetes is controversial and uncertain. This review is intended to help the reader understand the various GV metrics currently being reported in the literature, the potential mechanisms by which GV may contribute to the pathogenesis of the long-term complications of diabetes, and, finally, the evidence that reducing GV is beneficial to patients with type 1 diabetes.
\end{abstract}

\section{Keywords}

Continuous glucose monitoring (CGM), glycemic variability, mean amplitude of glycemic excursion (MAGE)

Disclosure: The software and methodology implemented in the 4 Diabetes Support System have been submitted to the US patent office, application number US60/901,703, and rights are co-owned by the Ohio University Technology Transfer Office, Cynthia R Marling, PhD, and Frank L Schwartz, MD, FACE.

Acknowledgments: The authors gratefully acknowledge the following sources of funding: National Science Foundation, Medtronic, Ohio University Russ College Biomedical Engineering Fund, J O Watson, DO Endowed Diabetes Research Chair of the Osteopathic Heritage Foundation, Ohio University Heritage College of Osteopathic Medicine Research and Scholarly Affairs Committee, and the Ohio University Diabetes Research Initiative.

Received: May 25, 2014 Accepted: June 2, 2014 Citation: US Endocrinology, 2014;10(1):20-4 DOI: 10.17925/USE.2014.10.01.20

Correspondence: Frank L Schwartz, MD, FACE, Professor of Endocrinology, J O Watson Chair for Diabetes Research, The Diabetes Institute, Ohio University Heritage College of Osteopathic Medicine, 331 Academic Research Center, Athens, OH 45701, US. E: schwartf@ohio.edu

One of the first observations that led to the consideration of glycemic variability (GV) as a potential contributor to diabetes complications in type 1 diabetes was from retrospective analysis of data from the Diabetes Control and Complications Trial (DCCT) study, which suggested that risk for retinopathy is determined by factors other than glycosylated hemoglobin $\left.\left(\mathrm{HbA}_{1 \mathrm{C}}\right)\right)^{1,2}$ In addition to elevated $\mathrm{HbA}_{1 \mathrm{C}}$ levels, fasting glucose levels, post-prandial (PP) hyperglycemia, total glucose exposure, and $\mathrm{HbA}_{1 \mathrm{c}}$ variability over time do correlate strongly with risk for complications in type 1 diabetes. ${ }^{2-5}$ Glycemic variability is defined as the fluctuation, or swings, of blood glucose levels between highs and lows. Of concern are abnormal excursions of blood glucose levels outside of the normal range seen in persons without diabetes. Excessive GV has been shown to be associated but not proven with increased mortality in critically ill patients with type 2 diabetes, ${ }^{6-9}$ as well as increased risk for both microvascular and macrovascular complications. ${ }^{10}$ However, the evidence that excessive GV (although the greater in type 1 diabetes) contributes to complications in type 1 diabetes is less convincing. ${ }^{11-14}$

Blood glucose levels in individuals who do not have diabetes typically range between $68-90 \mathrm{mg} / \mathrm{dl}$ before meals and rise to as high as $128 \mathrm{mg} / \mathrm{dl}$ following meals (90-180 minutes). Glucose levels are maintained very closely within this range in persons without diabetes through the complex interplay of multiple hormones but especially insulin. Glycemic variability tends to increase as insulin function and/ or levels decline, beginning with obesity and glucose intolerance (pre-diabetes), worsening with the onset of type 2 diabetes, and still greater in subjects with long-standing type 2 diabetes. ${ }^{15-18}$ Increasing GV in type 2 diabetes is a reflection of the progressive loss of residual beta cell function while in type 1 diabetes, insulin deficiency is the major abnormality from early onset, and exogenous replacement is required to control glucose levels thus GV is usually much greater from the onset. As our capacity to measure patient glucose levels more accurately, more reliably, and more frequently (i.e., continuous glucose monitoring [CGM]) has increased, our capacity to quantify GV data automatically in the clinical setting from CGM tracings has also improved. Figure 1 demonstrates two 24-hour CGM tracings in which one represents low GV and the other high GV. The capacity to routinely obtain 24-hour CGM tracings in patients has resulted in the reporting of many different indices of GV by numerous clinical investigators over the past 10 years as they have attempted to correlate GV with diabetes complication risk. The purpose of this review is to help the reader who does not actively participate in clinical research in this area of diabetes to understand all of the various GV metrics currently being reported in the literature, the possible mechanisms by which GV may contribute to the pathogenesis of the long-term complications of diabetes, and finally the evidence that reducing GV is actually beneficial to patients with type 1 diabetes without inducing harm. 


\section{Measures of Glycemic Variability}

various measures of GV have been developed to account for different aspects of GV that might contribute to tissue damage in individuals with diabetes including the period of time in which glucose levels are either above or below normal glucose levels (68-128 ng/dl), the frequency of the excursions, the rate of change of the excursions (slope), as well as the magnitudes of the excursions. Service et al. were the first to attempt the quantification of GV with their description, in 1970, of the mean amplitude of glycemic excursion (MAGE). ${ }^{19}$ MAGE measures the average heights of glucose excursions between peaks (highs) and nadirs (lows) that exceed the standard deviation (SD) for an individual within a given day. Since then, a large number (>50) of additional GV metrics have been proposed and extensively reviewed. ${ }^{17-22}$ Rodbard has categorized them into four distinct types: (1) methods related to $\mathrm{SD}$, such as interquartile range (IQR); (2) methods based on glucose excursions, such as MAGE; (3) methods based on day-to-day variability, such as the mean of daily differences (MODD); and (4) methods based on variability over relatively short time periods (hours), such as continuous overlapping net glycemic action (CONGA) ${ }^{20,21}$ Despite the large number of GV metrics that have been proposed, there is no agreed upon successor to MAGE among experts in this field of diabetes research.

Theadvent of CGMS with the capacity to reliably measure interstitial glucose levels every 1-5 minutes for 3-10 days at a time has led to automated systems that calculate MAGE as well as many other parameters of GV such as area under the curve (AUC), percent of time spent in the normal glucose target range, and percent of time spent above or below the target range. We recently developed an automated system to calculate GV that determines 24-hour values for MAGE, SD, as well as three new metrics. The new metrics are: distance traveled (DT), which captures overall fluctuation and is similar to mean absolute glucose (MAG), ${ }^{23}$ excursion frequency (EF), the number of glucose excursions outside the normal range:22 and the consensus perceived glycemic variability (CPGV). ${ }^{23}$ Consensus perceived glycemic variability is a metric that does not fit into any of Rodbard's categories. Rather, it integrates multiple facets of GV via machine learning algorithms and is based on physician perception of GV. The notion is one suggested to us by David Klonoff, MD, that 'physicians know glycemic variability when they see it.' Using over 250 individual 24hour CMG plots, we had 12 diabetes experts from around the world rate each one on a GV scale: Iow (1), borderline (2), high (3), and extremely high (4). We then averaged all responses to each plot and from that developed consensus physician GV ratings ranging from 1 to 4 . Figure 2 shows how MAGE, EF, and DT are determined, in panels $A, B$, and $C$, respectively. Panel D illustrates representative scores for MAGE, EF, DT, and CPGV on two different tracings with different magnitudes of GV. The CPGV, when applied to blinded CGM plots, produced a more accurate, sensitive, and specific determination of excessive GV than EF, DT, SD, or MAGE. ${ }^{24}$ Figure 3 is an illustration of how the daily and 2-week average CPGV could be reported to the clinician at a routine clinical appointment. Although this new metric seems promising, it needs to be stressed that at this time there is no gold standard for assessing GV and only conflicting data supporting the value of GV in predicting complications risk in patients with type 1 diabetes. A recent review of four automated programs previously reported to calculate GV demonstrated variable agreement on routine calculations such as MAGE. ${ }^{24}$

\section{Figure 1: Two 24-hour Continuous Glucose Monitoring Tracings Representing High Glycemic Variability (GV) (A) and Low GV (B)}
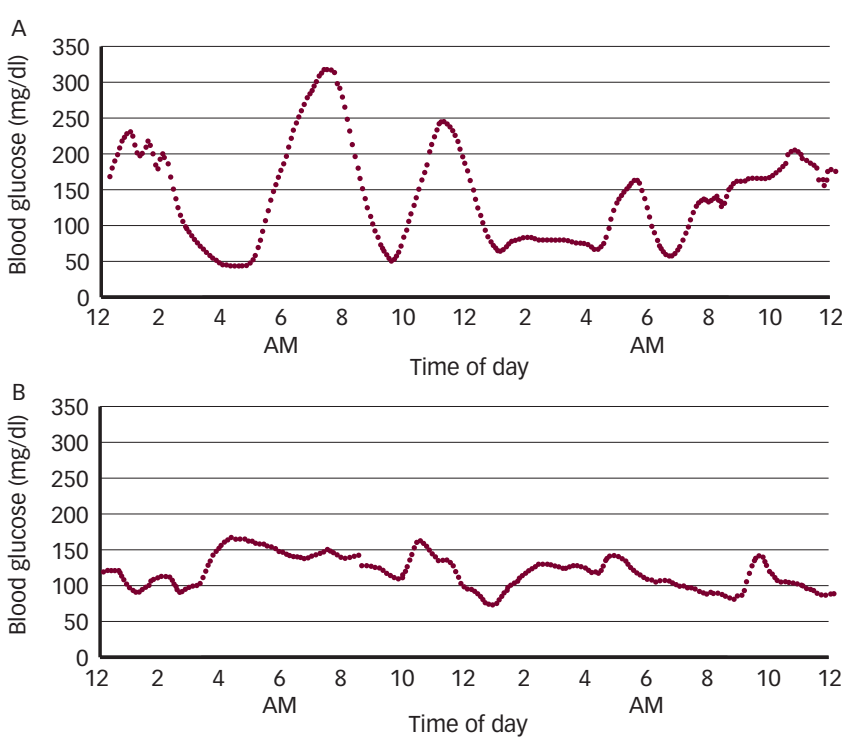

\section{Figure 2: Determining Mean Amplitude of Glycemic Excursion, Excursion Frequency, and Distance Traveled and their Application on Continuous Glucose Monitoring Tracings}
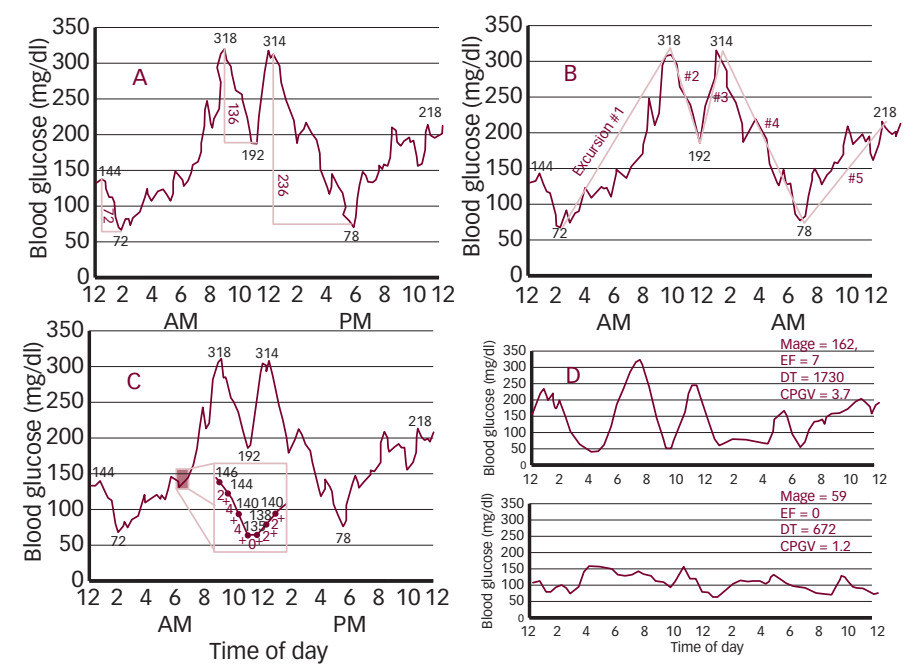

The figure shows how mean amplitude of glycemic excursion (MAGE), excursion frequency $(E F)$, and distance traveled (DT) are determined, in panels $A, B$, and $C$, respectively. Panel D illustrates representative scores for MAGE, EF, DT, and consensus perceived glycemic variability (CPGV) on two different continuous glucose monitoring (CGM) tracings with different magnitudes of GV.

\section{Glycemic Variability and Mechanisms of Tissue Damage}

The mechanisms by which chronic hyperglycemia induces diabetes complications are well established while the mechanisms for induction of tissue damage by GV are less substantiated and these will be described. Figure 4 illustrates probable mechanisms for both processes. Sustained hyperglycemia results in end-organ damage through the accumulation of non-metabolizable sugars in certain cells and the non-covalent 


\section{Figure 3: How Daily and 2-week Average Consensus Perceived Glycemic Variability could be Reported to the Clinician at a Routine Clinical Appointment}

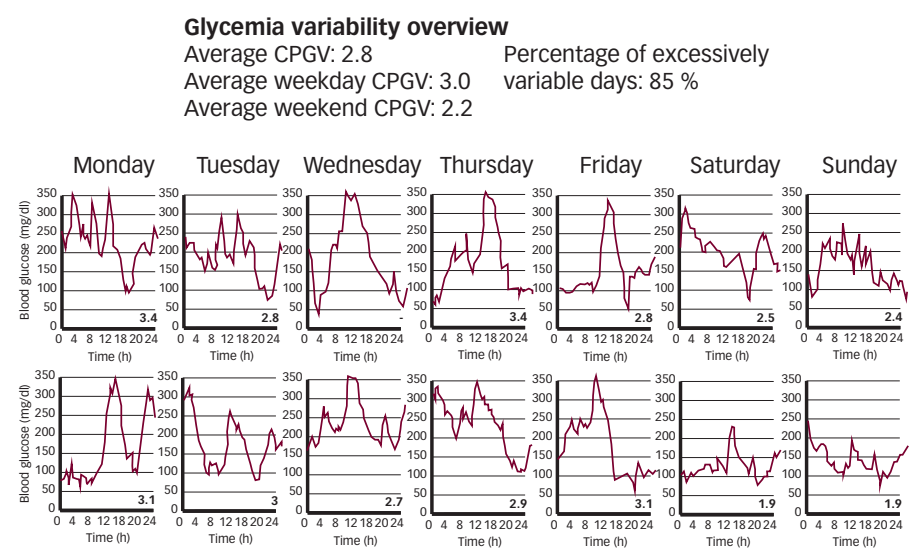

CPGV = consensus perceived glycemic variability.

\section{Figure 4: Mechanisms of Diabetes Complications}

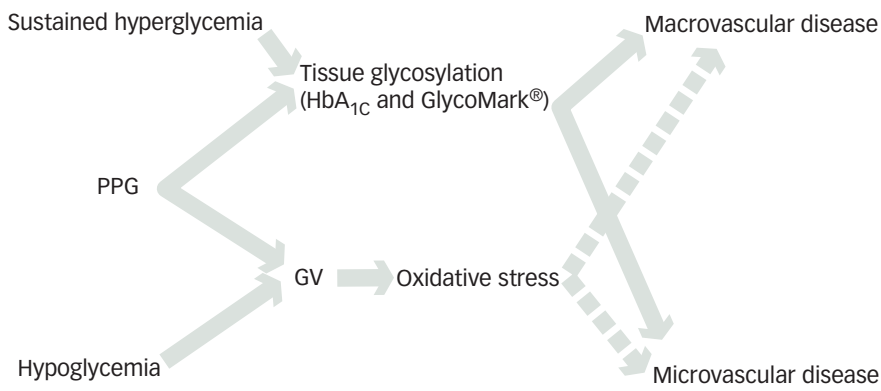

$G V=$ glycemic variability; $H D A_{1 c}=$ glycosylated hemoglobin; $P P G=$ post-prandial hyperglycemia .

bonding of glucose (glycosylation) to multiple tissue proteins in others. Mechanisms include: (1) the Poly-ol Pathway in non-insulin requiring tissues such as nerve and lens where glucose crosses the cell membrane against a concentration gradient. Intracellularly, the glucose is converted into non-metabolizable sugars such as fructose and sorbitol which then accumulate, induce cellular osmotic changes resulting in water being drawn into the cell as well as the depletion of key intracellular components in the cells such as myoinositol and nicotinamide adenine dinucleotide phosphate (NADPH) as the cells try to normalize the osmotic differences, ${ }^{25}$ (2) glycation of key proteins such as collagen with resultant changes in their tertiary structure leading to tissue and organ damage; ${ }^{26}$ (3) formation of advanced glycosylated end-products (AGES), ${ }^{27}$ which trigger multiple inflammatory cytokine and growth pathways in target cells; ${ }^{28}$ and each of these effects can result in (4) activation of protein kinase $C-\beta$ (PKC- $\beta$ ), mitogen-activated protein kinase (MAPK), and nuclear factor kappa $B(N F-K \beta$ ), which result in activation and induction of both oxidative stress, ${ }^{29}$ and other inflammatory cytokine pathways leading to vascular, immunological, and direct end-organ tissue damage. ${ }^{30}$

The effect of GV is more complex to assess and separate from tissue glycosylation when trying to explain its relationship to long-term diabetes complications. Tissue glycation is primarily a time and glucose concentrationdependent process. By contrast, changes in glucose concentrations included in a GV metric would have to include the magnitude and frequency of each hyperglycemic and hypoglycemic episode within a specific period of time (24 hours), as well as the length of time of each hyperglycemic and hypoglycemic event. GV is thought to damage endothelial cells through the induction of oxidative stress. ${ }^{31,32}$ However, acute changes in glucose concentrations can also directly damage neural (brain) tissue if they are high or low enough. ${ }^{33,34}$ Monnier was the first to demonstrate that glucose fluctuations induced greater oxidative stress (as measured by 24-hour urine 8-iso PGF2 alpha [PGF2]) compared with chronic hyperglycemia in patients with type 2 diabetes. ${ }^{35}$ However, in one review of the literature concerning the effect of GV on long-term complications risk in type 1 diabetes, only two of eight papers demonstrated any relationship between diabetes complications and only one of eight used CGM to calculate GV.12,14 One of the major problems with GV calculations is that their values typically only reflect a 24-hour period or less while $\mathrm{HbA}_{1 \mathrm{C}}$ is a measure of the past 6-8 weeks of hyperglycemia and GlycoMark ${ }^{\circledR}$ (see later description) is a measure of the past 2-3 weeks. To emphasize this point, the Epidemiology of Diabetes Interventions Complications (EDIC) study from the DCCT revealed that variations in $\mathrm{HbA}_{1 \mathrm{C}}$ over time were a very strong predictor of microvascular complications ${ }^{2}$ while a 24-hour urine PGF2 is only a reflection of one day's oxidative stress.

A final component of GV contributing to tissue damage and complications from diabetes that also is not captured by $\mathrm{HbA}_{1 \mathrm{C}}$ or GlycoMark is hypoglycemia. Hypoglycemia if present in a given patient is known to reduce the average $\mathrm{HbA}_{1 \mathrm{c}}$ of that patient, which underestimates the impact of hyperglycemia on complication risk. Yet, elevated $\mathrm{HbA}_{1 \mathrm{c}}$ and high $\mathrm{GV}$ together in the same patient are associated with increased frequency and severity of hypoglycemic episodes in both forms of diabetes, and increased GV has been shown to precede episodes of hypoglycemia in CGM data. ${ }^{36-38}$ Recurrent hypoglycemia blunts the counter-regulatory response to the next hypoglycemic event resulting in more frequent and severe episodes of hypoglycemia. ${ }^{8}$ In children and adolescents with type 1 diabetes, in whom insulin-induced hypoglycemia was intentionally induced (blood glucose $<60 \mathrm{ng} / \mathrm{dl}$ ) in a clinical research center setting, elevated GV and CONGA calculated from 3 days of CMG prior to the insulin-induced hypoglycemia were indicators of an impaired glucagon response to the event which points to a mechanism of hypoglycemia begetting hypoglycemia. ${ }^{36}$ Finally, hypoglycemia is associated with increased mortality in the intensive care unit (ICU) setting. ${ }^{39}$

Hypoglycemia contributes to long-term complications by processes different from hyperglycemia. Studies have demonstrated that hypoglycemia induces both proinflammatory and prothrombotic pathways in addition to oxidative stress in endothelial tissues. ${ }^{40-42}$ These are the probable mechanisms for the increased risk for vascular events in intensively treated $\left(\mathrm{HbA}_{1 \mathrm{C}}<7 \%\right)$, older type 2 diabetes patients with pre-existing cardiovascular complications reported in the NICE-SUGAR study ${ }^{43}$ and in patients with acute myocardial infarction who developed hypoglycemia during attempted intensive glucose control. ${ }^{44}$

How a particular tissue, organ, or individual patient responds to hypoglycemia itself is another variable which has not been adequately studied in the context of GV in patients with type 1 diabetes other than acute neurological effect or long-term impairment of cognitive function. Other studies looking at mechanisms of hypoglycemia-induced vascular 
complications include clamp studies of insulin-induced hypoglycemia in both normal and type 1 diabetes patients, which have been shown to induce the release of adhesion molecules (intercellular adhesion molecule [ICAM], vascular cell adhesion molecule [VCAM], E-Selectin, P-Selectin), inflammatory cytokines (tumor necrosis factor-alpha [TNF- $\alpha$ ], interleukin 6 [IL-6]), and pro-atherogenic (plasminogen activator inhibitor-1 [PAI-1], vascular endothelial growth factor [VEGF]) factors..$^{40,45}$ In these studies the physiological responses to the hypoglycemia were both concentration and time-dependent. Therefore, we feel that future studies looking at the relationship between GV and risk for diabetes complications should measure markers of oxidative stress, inflammation, coagulation, and endothelial function to capture the effects of hypoglycemia.

\section{Clinical Markers of Inflammation, Excessive Oxidative Stress, and Glycemic Variability in Type 1 Diabetes}

Results from studies looking at biochemical and physiological markers of GV in patients with type 1 diabetes have produced conflicting data. Table 1 lists the common markers that have been assessed in clinical trials in addition to $\mathrm{HbA}_{1 \mathrm{c}}$ trying to link GV with tissue damage and long-term diabetic complications. GlycoMark (1,5-anhydroglucitol or 1, 5-AG2) ${ }^{46}$ is a naturally occurring monosaccharide found in nearly all foods and is filtered in the blood like glucose but competes with glucose in the renal proximal tubule, which decreases during times of hyperglycemia above $180 \mathrm{mg} / \mathrm{dl}$ and cannot be reabsorbed. Thus, in periods of hyperglycemia, serum 1,5AG levels drop, and so are inversely correlated with high glucose levels. 1,5-AG levels are now used as a measure of post-prandial hyperglycemia in patients with high GV that may not be detected with $\mathrm{HbA}_{1 c^{4}}{ }^{46-48}$ However, 1,5-AG does not show significant correlation with measures of CONGA-1, MAGE, and MODD or markers of oxidative stress in patients with type 2 diabetes. ${ }^{49}$ Measurement of 24-hour urinary PGF2 is commonly used as a surrogate marker of daily oxidative stress when obtained as a 24-hour measurement compared to assessments of GV.50 Isoprostanes are stable end-products of lipid peroxidation and are commonly and easily measured in human urine. Higher levels of urine PGF2 correlate quite well with GV and with risk for macrovascular complications in patients with type 2 diabetes ${ }^{35}$ in multiple studies as well as survival in the ICU. However, adolescents and adults with type 1 diabetes exhibit much higher levels of GV and 24-hour urinary PGF2 compared with non-diabetics and individuals with type 2 diabetes. ${ }^{13}$ High GV was also observed in children and adolescents attending a summer camp, where no correlations were found between GV and urinary PGF2. ${ }^{51}$ Other studies have also reported the lack of correlation between increased GV and urine PGF2 in type 1 diabetes. Additional markers of oxidative stress have been measured in patients with type 1 diabetes with significant correlations between elevated serum levels of VEGF, nitrous oxide (NO), and malondialdehyde (MDA) in individuals with poorly controlled diabetes, however, GV was not assessed. Studies looking at the adhesion molecules (ICAM, VCAM, E-Selectin, P-Selectin), inflammatory cytokines (TNF- $\alpha, I L-6$ ), and pro-atherogenic (PAI-1 or VEGF) factors have also been seen in patients with uncontrolled type 1 diabetes but GV has not been assessed in these studies. A recent report assessing the relationship between single nucleotide polymorphisms (SNPS) of 15 oxidative stress genes which were combined into an 'oxidative stress risk allele score' and atherosclerosis was predicted using this score and demonstrated a 1.5fold risk increase per abnormal allele $(p<0.001)$. The combined risk alleles were also associated with elevated plasma myeloperoxidase (MPO) levels

\section{Table 1: Markers of Risk for Diabetes Complications}

- Glycosylated hemoglobin ( $\left.\mathrm{HbA}_{1 \mathrm{C}}\right)$

-1,5-anhydroglucitol (AG)

- 8-iso-PGF2 alpha (PGF2)

- Nitrous oxide (NO)

- Malondialdehyde (MDA)

suggesting that testing for genetic markers of oxidative stress or induction of certain genes might be a future target seeking to link GV with micro and macrovascular risk in type 1 diabetes.

A physiological study measuring carotid intima medial thickness and vessel stiffness in patients with type 1 diabetes recently demonstrated significant correlations with age, blood pressure, heart rate, and daily insulin intake but no statistically significant correlation with MAGE or 'post-prandial glucose spikes' was observed. However, this study again did not look at additional markers of complication risk such as GV.

\section{Clinical Evidence that Glycemic Variability Contributes to Complications in Type 1 Diabetes}

Analysis of data from the EDIC study to see whether longer-term followup of DCCT patients suggested that 'glycemic instability' contributed to risk for later retinopathy and nephropathy as fasting glucose levels, post-prandial glucose levels, and $\mathrm{HbA}_{1 c}$ correlated strongly with risk for later complication. ${ }^{1,2,4}$ In the EDIC publication, GV calculated from daily 5-7 fingerstick glucose data points using SD or MAGE as the indices failed to predict risk. ${ }^{5}$ Interestingly, these same investigators demonstrated that long-term glucose variability as defined by 'variable $\mathrm{HbA}_{1 \mathrm{c}}$ ' levels over the years did contribute to development of retinopathy and nephropathy in these type 1 diabetes patients. ${ }^{2}$ However GV parameters calculated from 5-7 fingerstick glucose levels per day may have been inadequate metrics of GV to detect GV's contribution to complication risk in type 1 diabetes in the DCCT. ${ }^{13}$ As previously mentioned, adolescents and adults with type 1 diabetes exhibit higher measures of GV and higher levels of 24-hour urinary PGF2 compared with adults with type 2 diabetes and non-diabetics. ${ }^{13}$ Recent studies of type 1 diabetes patients using CGM data to calculate GV to assess risk for complications are beginning to show significant correlation for risk. One study demonstrated that type 1 diabetes patients with any evidence of early microvascular complications (retinopathy, microalbuminuria, neuropathy) had significantly higher GV than patients with comparable glycemic control $\left(\mathrm{HbA}_{1 \mathrm{c}}\right)$ without complications. ${ }^{52}$ Other studies have shown correlation between GV (3-day mean glucose MG levels) and endothelial function (as measured by assessing post-occlusion forearm vascular resistance) $)^{53}$ while another study in adolescents with type 1 diabetes undergoing fitness testing demonstrated an inverse relationship between mean $\mathrm{VO}_{2}$ max and MAGE as well as an inverse relationship between metabolic work load (measure of total accumulated workload during exercise) and MAGE. ${ }^{54}$ These limited, preliminary studies do support the concept that individuals with type 1 diabetes and high GV are experiencing physiological changes that can result in tissue/end-organ damage (endothelial cells), these effects can now be measured both biochemically and physiologically, and confirmatory studies are on the horizon. 


\section{Clinical Significance of Type 1 Diabetes Glycemic Variability in 2014}

Just as older physicians knew intuitively that hyperglycemia contributed to the long-term complications of diabetes, so do current physicians intuitively 'know' that GV contributes to both short-and long-term complications. The relationship between high GV and the increased risk for hypoglycemia in both forms of diabetes at all stages of life is generally accepted. $6,36,37,55,56$ From a safety perspective, all patients with high GV and hypoglycemia should initially have 'reverse intensification' of therapy, lowering of insulin doses, and elimination of the lows before restarting attempts to improve overall glucose levels. ${ }^{38}$ Currently, there are no recommendations to target high GV in patients with type 1 diabetes as a means of reducing complication risk. However, now that various parameters of GV can be calculated automatically, we feel that future prospective, multisite outcomes studies measuring agreed upon parameters of GV by CGM, as well as serological markers of tissue endothelial inflammation and oxidative stress, are ultimately required to turn a clinical hunch into a certainty.
1. Kilpatrick ES, Rigby AS, Atkin SL, The effect of glucose variability on the risk of microvascular complications in type 1 diabetes, Diabetes Care, 2006:29:1486-90.

2. Kilpatrick ES, Rigby AS, Atkin SL, A1C variability and the risk of microvascular complications in type 1 diabetes: data from the Diabetes Control and Complications Trial, Diabetes Care 2008;31:2198-202

3. Ceriello A, Postprandial hyperglycemia and diabetes complications: is it time to treat? Diabetes, 2005;54:1-7.

4. Kilpatrick ES, Rigby AS, Atkin SL, Mean blood glucose compared with $\mathrm{HbA} 1 \mathrm{C}$ in the prediction of cardiovascular disease in patients with type 1 diabetes, Diabetologia, 2008:51:365-71.

5. Lachin JM, Genuth S, Nathan DM, et al., Effect of glycemic exposure on the risk of microvascular complications in the diabetes contro and complications trial--revisited, Diabetes, 2008;57:995-1001.

6. Egi M, Bellomo R, Stachowski E, et al., Variability of blood glucose concentration and short-term mortality in critically ill patients, Anesthesiology, 2006;105:244-52.

7. Krinsley JS, Glycemic variability: a strong independent predictor of mortality in critically ill patients, Crit Care Med, 2008:36:3008-13.

8. Moore J, Dungan K, Glycemic variability and glycemic control in the acutely ill cardiac patient, Heart Fail Clin, 2012;8:523-38.

9. Hermanides J, Vriesendorp TM, Bosman RJ, et al., Glucose variability is associated with intensive care unit mortality, Crit Care Med, 2010;38:838-42

10. Muggeo M, Zoppini $G$, Bonora $E$, et al., Fasting plasma glucose variability predicts 10 -year survival of type 2 diabetic patients: the Verona Diabetes Study Diabetes Care, 2000;23:45-50.

11. Monnier $L$, Colette $C$, Leiter $L$, et al., The effect of glucose variability on the risk of microvascular complications in type 1 diabetes, Diabetes Care, 2007;30:185-6; author reply 187-8.

12. Nalysnyk L, Hernandez-Medina M, Krishnarajah G, Glycemic variability in patients with diabetes mellitus: evidence from a systemic review of the literature, Diabetes Obes Metab 2010;12:288-98.

13. Wentholt IM, Kulik W, Michels RP, et al., Glucose fluctuations and activation of oxidative stress in patients with type 1 diabetes, Diabetologia, 2008:51:183-90.

14. Hinzmann R, Schlaeger C, Tran CT, What do we need beyond hemoglobin A1C to get the complete picture of glycemia in people with diabetes? Int J Med Sci, 2012;9:665-81.

15. Madhu SV, Muduli SK, Avasthi R, Abnormal glycemic profiles by CGMS in obese first-degree relatives of type 2 diabetes mellitus patients, Diabetes Technol Ther, 2013:15:461-5.

16. Kohnert KD, Heinke P, Fritzsche G, et al., Evaluation of the mean absolute glucose change as a measure of glycemic variability using continuous glucose monitoring data, Diabetes Technol Ther, 2013;15:448-54.

17. Rodbard $D$, Increased glycemic variability at the onset and during progression of type 2 diabetes-commentary, Diabetes Technol Ther, 2013;15:445-7.

18. Chen T, XU F, Su JB, et al., Glycemic variability in relation to oral disposition index in the subjects with different stages of glucose tolerance Diabetol Metab Syndr 2013:5:38-47.

19. Service FJ, Molnar GD, Rosevear JW, et al., Mean amplitude of glycemic excursions, a measure of diabetic instability, Diabetes, 1970;19:644-55

20. Rodbard D, New and improved methods to characterize glycemic variability using continuous glucose monitoring,
Diabetes Technol Ther, 2009;11:551-65.

21. Rodbard D, Interpretation of continuous glucose monitoring data: glycemic variability and quality of glycemic control, Diabetes Technol Ther, 2009:11(Suppl. 1):S55-67.

22. Marling CR, Shubrook JH, Vernier SJ, et al., Characterizing blood glucose variability using new metrics with continuous glucose monitoring data, J Diabetes Sci Technol, 2011;5:871-8.

23. Marling CR, Struble NW, Bunescu RC, et al., A consensus perceived glycemic variability metric, J Diabetes Sci Technol, 2013;7:871-9.

24. Sechterberger MK, Luiif YM, Devries JH, Poor agreement of computerized calculators for mean amplitude of glycemic excursions, Diabetes Technol Ther, 2014:16:72-5.

25. Barnett PA, González RG, Chylack LT Jr, Cheng HM, The effect of oxidation on sorbitol pathway kinetics, Diabetes, 1986;35:426-32

26. Ulrich $\mathrm{P}$, Cerami $\mathrm{A}$, Protein glycation, diabetes, and aging, Recent Prog Horm Res, 2001;56:1-21.

27. Yamagishi S, Role of advanced glycation end products (AGES) and receptor for AGES (RAGE) in vascular damage in diabetes, Exp Gerontol, 2011:46:217-24.

28. Sytze Van Dam P, Cotter MA, Bravenboer B, Cameron NE, Pathogenesis of diabetic neuropathy: focus on neurovascular mechanisms, Eur J Pharmacol, 2013;719:180-6.

29. Cameron NE, Cotter MA, Effects of protein kinase cbeta inhibition on neurovascular dysfunction in diabetic rats: interaction with oxidative stress and essential fatty acid dysmetabolism, Diabetes Metab Res Rev, 2002;18:315-23.

30. Tarr JM, Kaul K, Chopra M, et al., Pathophysiology of diabetic retinopathy, ISRN Ophthalmol, 2013;343560.

31. Ceriello A, Ihnat MA, Oxidative stress is, convincingly, the mediator of the dangerous effects of glucose variability, Diabet Med, 2010;27:968

32. Ceriello A, Ihnat MA, 'Glycaemic variability': a new therapeutic challenge in diabetes and the critical care setting, Diabet Med, 2010;27:862-7.

33. Wright RJ, Newby DE, Stirling D, et al., Effects of acute insulininduced hypoglycemia on indices of inflammation: putative mechanism for aggravating vascular disease in diabetes, Diabetes Care, 2010;33:1591-7.

34. Efron $\mathrm{D}$, South $\mathrm{M}$, Volpe JJ, Inder T, Cerebral injury in association with profound iatrogenic hyperglycemia in a neonate, Eur J Paediatr Neurol, 2003;7:167-71.

35. Monnier L, Mas E, Ginet C, et al., Activation of oxidative stress by acute glucose fluctuations compared with sustained chronic hyperglycemia in patients with type 2 diabetes, JAMA 2006:295:1681-7.

36. Alghothani N, Dungan KM, The effect of hypoglycemia on counterregulatory homone response to hypoglycemia in adolescents and children with type 1 diabetes, Diabetes Techno Ther, 2011;13:1085-9.

37. Kovatchev BP, Cox DJ, Farhy LS, et al., Episodes of severe hypoglycemia in type 1 diabetes are preceeded and followed within 48 hours by measurable disturbances in blood glucose, I Clin Endocrinol Metab, 2000:85:4287-92.

38. Cryer PE, Mechanisms of hypoglycemia-associated autonomic failure and its component syndromes in diabetes, Diabetes, 2005; 54:3592-601.

39. Hermanides J, Bosman RJ, Vriesendorp TM, et al., Hypoglycemia is associated with intensive care unit mortality, crit Care Med.
2010;38:1430-4

40. Gogitidze Joy N, Hedrington MS, Briscoe VJ, et al., Effects of acute hypoglycemia on inflammatory and pro-atherothrombotic biomarkers in individuals with type 1 diabetes and healthy individuals. Diabetes Care, 2010;33:1529-35.

41. Wieczorek I, Pell AC, Mclver B, et al., Coagulation and fibrinolytic systems in type I diabetes: effects of venous occlusion and insulin-induced hypoglycaemia, Clin Sci (Lond), 1993;84:79-86.

42. Ceriello A, Novials A, Ortega E, et al., Evidence that hyperglycemia after recovery from hypoglycemia worsens endothelial function and increases oxidative stress and inflammation in healthy control subjects and subjects with type 1 diabetes, Diabetes, 2012:61:2993-7.

43. NICE-SUGAR Study Investigators, Finfer S, Chittock DR, Su SY, et al., Intensive versus conventional glucose control in critically ill patients, N Eng/ J Med, 2009;360:1283-97.

44. Kosiborod M, Inzucchi SE, Goyal A, et al., Relationship between spontaneous and iatrogenic hypoglycemia and mortality in patients hospitalized with acute myocardial infarction, JAMA 2009:301:1556-64

45. Razavi Nematollahi L, Kitabchi AE, Stentz FB, et al., Proinflammatory cytokines in response to insulin-induced hypoglycemic stress in healthy subjects, Metabolism, 2009;58:443-8.

46. Goto M, Yamamoto-Honda R, Shimbo T, et al., Correlation between baseline serum 1,5-anhydroglucitol levels and 2-hour post-challenge glucose levels during oral glucose tolerance tests, Endocr J, 2011:58:13-7.

47. Dungan $\mathrm{KM}, 1,5$-anhydroglucitol (GlycoMark) as a marker of short-term glycemic control and glycemic excursions, Expert Rev Mol Diagn, 2008:8:9-19.

48. Juraschek SP, Steffes MW, Selvin E, Associations of alternative markers of glycemia with hemoglobin $\mathrm{A}(1 \mathrm{C})$ and fasting glucose Clin Chem, 2012;58:1648-55

49. Kim TH, Choi SE, Ha ES, et al., IL-6 induction of TLR-4 gene expression via STAT3 has an effect on insulin resistance in human skeletal muscle, Acta Diabetol, 2013;50:189-200.

50. Morrow JD, Hill KE, Burk RE, et al., A series of prostaglandin F2-like compounds are produced in vivo in humans by a noncyclooxygenase, free radical-catalyzed mechanism, Proc Natl Acad Sci U S A, 1990;87:9383-7.

51. Colomo N, Tapia MJ, Vallejo MR, et al., Glycemic variability and oxidative stress in children, with type 1 diabetes attending a summer camp, An Pediatr (Barc), 2013:9:1-7.

52. Soupal J, Skrha J Jr, Fajmon M, et al., Glycemic variability is higher in type 1 diabetes patients with microvascular complications irrespective of glycemic control, Diabetes Technol Ther, 2014;16:198-203.

53. Hoffman RP, Dye AS, Huang H, Bauer JA, Effects of glucose control and variability on endothelial function and repair in adolescents with type 1 diabetes, ISRN Endocrinol, 2013;876547.

54. Singhvi A, Tansey $\mathrm{M}$, Janz $\mathrm{M}$, et al., Aerobic fitness and glycemic variability in adolescents with type 1 diabetes, Endocr Pract, 2014:21:1-18.

55. Egi M, Bellomo R, Reade MC, Is reducing variability of blood glucose the real but hidden target of intensive insulin therapy? Crit Care, 2009;13:302

56. Arnold P, Paxton RA, MCNorton $K$, et al., The effect of a hypoglycemia treatment protocol on glycemic variability in critically ill patients, J Intensive Care Med, 2013:Epub ahead of print. 